\title{
Collagen from marine source for regenerative therapy: A literature review
}

Cite as: AIP Conference Proceedings 2314, 050017 (2020); https://doi.org/10.1063/5.0036110 Published Online: 09 December 2020

\section{Hadyan Farizan Zata, Prahasanti Chiquita, and Kurnia Shafira}

\section{ARTICLES YOU MAY BE INTERESTED IN}

Effectiveness of silver nanoparticles synthesized using Diospyros discolor Willd. (Bisbul) leaf extract for antimicrobial agents

AIP Conference Proceedings 2314, 020007 (2020); https://doi.org/10.1063/5.0034464

Ball detection for KRSBI soccer robot using PeleeNet on omnidirectional camera AIP Conference Proceedings 2314, 040012 (2020); https://doi.org/10.1063/5.0036172

Titanium implant coating and their effect on osseointegration AIP Conference Proceedings 2314, 050018 (2020); https://doi.org/10.1063/5.0036187

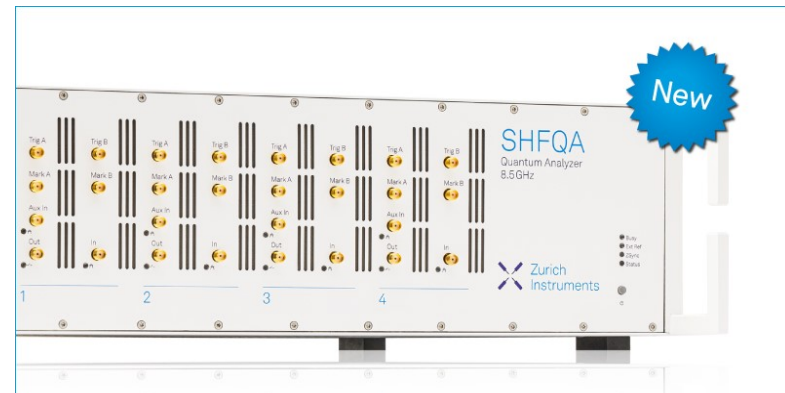

\section{Your Qubits. Measured.}

Meet the next generation of quantum analyzers

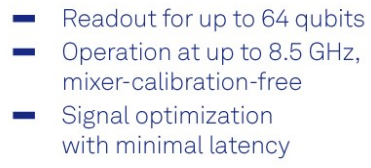




\title{
Collagen from Marine Source for Regenerative Therapy: a Literature Review
}

\author{
Hadyan Farizan Zata ${ }^{1, \text { a) }}$, Prahasanti Chiquita ${ }^{1, b)}$, Kurnia Shafira ${ }^{1}$ \\ ${ }^{1}$ Department of Periodontology, Faculty of Dental Medicine,Airlangga University, Surabaya 60132, Indonesia. \\ a) Corresponding author: rizanzata@rocketmail.com \\ b) chiquita-p-s@fkg.unair.ac.id
}

\begin{abstract}
All species of multicellular organisms are formed from connective tissue in which the main forming protein is collagen. Collagen is usually extracted from animal species and their waste products. Because of the prevalence of dangerous infectious diseases (zoonosis) and religious reasons in some countries, research is needed on other sources of non-mammalian natural biomaterials. At present, many bioactive material derived from marine source have been developed. Collagen derived from marine source has several advantages, namely good biocompatibility, low imunogenicity, high levels of direct cell adhesion, and high biodegradability. The excellent biocompatibility of marine collagen has shown its potential in tissue engineering and in regenerative therapy for biomaterial scaffolding.
\end{abstract}

\section{INTRODUCTION}

Silvestri et al have reviewed biomaterial (polymers) comprehensively including collagen, gelatin, chitosan, hyaluronic acid, matrigel, fibrin, cellulose, alginate, and silk fibers [1]. Collagen is found in various forms in the tissue of all species of multicellular organisms, this is a major element of the extracellular matrix (ECM), and performs various functions depending on its location $[2,3]$. Various animal species and their waste products can be extracted into collagen which is bones, tendons, cartilage, and skin [4]. Several studies of collagen from natural sources, such as bovine and porcine have conventionally been used in biomedical applications [5]. Because of the regenerative nature of the collagen, this is further applied to regenerative medicine [6].

Nowadays, the problem of using collagen from mammalian sources is the infectious diseases (zoonosis) such as avian influenza and swine flu, spongiform bovine encephalopathy, teeth-and mouth disease in bovine, porcine, and buffalo, Ebola, and Zika fever [7]. The use of collagen from mammalian sources is also limited in some countries due to religious and cultural reasons. Therefore, an alternative source of collagen is needed. Bioactive material derived from the sea, fish recently attracted attention with low immunogenic collagen properties and few secondary complications in the human body. Fish collagen extracted from scales, skin, and bones, and has good bioactive properties, such as good biocompatibility, high biodegradability, low imunogenicity, and potential of cell proliferation $[8,9]$. Collagen from marine sources has the disadvantage of low denaturation temperature (Td), this shows poor stability. However, with the cross-linking method this can be overcome [10].

\section{COLLAGEN AS SCAFFOLD MATERIAL IN REGENERATIVE THERAPY}

Collagen is part of a protein that is very characteristic and often found in multicellular organism and it is a protein that is most often found in mammals. This makes collagen the most widely studied biomaterials from extracellular matrix (EMC). $30 \%$ of the human body is collagen which is useful as a provider of texture, shape and resistance and is the most commonly found in connective tissue in animal. Bones, dermis, cartilage (hyaline), ligaments, tendons, teeth, blood vessels, cornea, intervertebral discs, vitreous body, and placenta (fetus) is where the collagen most found. Based on the primary structure or form of organization and function, collagen can be classified and available as many

The 2nd International Conference on Physical Instrumentation and Advanced Materials 2019

AIP Conf. Proc. 2314, 050017-1-050017-7; https://doi.org/10.1063/5.0036110

Published by AIP Publishing. 978-0-7354-4056-2/\$30.00 
as 29 types of collagen have been found. Because of its various structural and functional properties, collagen is a significant part of tissue and biomedical engineering.

Collagen fibers are formed from collagen types I, II, III, V and XI. There are $3 \alpha$ chains in the collagen molecule and form its molecular arrangement. In each of these $\alpha$ chains, there are a thousand amino acids that have the -Gly-XY sequence. Glycine that found in these chains has a very important role in positioning the third amino acid that functions as attachment to the three $\alpha$ chains in the tropocollagen molecule, proline and 4-hydroxyproline most found in the $\mathrm{X}$ and $\mathrm{Y}$ sequence. The current major gold standard in tissue engineering is type I collagen. This type I collagen is chosen as an implantation material in a person because only a few people have an allergic reaction to this biomaterial and can be ascertained through a serological test.

Collagen is the most common protein that found on the earth because it can be extracted from various sources. Every living creature can be found in this collagen. Although many sources of collagen are obtained, but generally the materials most often used in tissue engineering applications are cow and tendon skin, pig skin, and mice. Marine biota also contributes very large collagen, such as extra collagen obtained from fish, jellyfish, and sponges.

Only a few of this collagen is used in research and clinical use, but it is already widely used in industry. Variety of fundamental applications has founded in vitro and in vivo in the use of collagen-based biomaterials from the collagen or acellular matrix that has been extracted. In today's modern techniques, bone and cartilage reconstruction is the most important part in functional and aesthetic surgery. When osteochondral defects occur that have reached important volumes and autograft must be avoided for pathological or practical reason, implantation of this collagenbased biomaterial is necessary [11].

TABLE 1. Types, forms, and distribution of collagen [11]

\begin{tabular}{|c|c|c|c|c|}
\hline & Type & Molecular formula & Polymerized form & Tissue distribution \\
\hline \multirow{5}{*}{$\begin{array}{l}\text { Fibril- } \\
\text { Forming } \\
\text { (fibrillar) }\end{array}$} & I & {$[\alpha 1(\mathrm{I})]_{2} \alpha 2(\mathrm{I})$} & fibril & $\begin{array}{l}\text { bone, skin, tendons, ligaments, } \\
\text { cornea (represent } 90 \% \text { of total } \\
\text { collagen of the human body) }\end{array}$ \\
\hline & II & {$[\alpha 1(\mathrm{II})]_{3}$} & fibril & $\begin{array}{l}\text { cartilage, intervertebrate disc, } \\
\text { notochord, vitreous humor in } \\
\text { the eye }\end{array}$ \\
\hline & III & {$[\alpha 1(\mathrm{III})]_{3}$} & fibril & skin, blood vessels \\
\hline & V & $\begin{array}{l}{[\alpha 1(\mathrm{~V})]_{2} \alpha 2(\mathrm{~V}) \text { and }} \\
\alpha 1(\mathrm{~V}) \alpha 2(\mathrm{~V}) \alpha 3(\mathrm{~V})\end{array}$ & $\begin{array}{c}\text { fibril } \\
\text { (assemble with } \\
\text { type I) }\end{array}$ & idem as type I \\
\hline & $\mathrm{XI}$ & $\alpha 1(\mathrm{XI}) \alpha 2(\mathrm{XI}) \alpha 3(\mathrm{XI})$ & $\begin{array}{c}\text { fibril } \\
\text { (assemble with } \\
\text { type II) }\end{array}$ & idem as type II \\
\hline \multirow{2}{*}{$\begin{array}{l}\text { Fibril- } \\
\text { associated }\end{array}$} & IX & $\alpha 1(\mathrm{IX}) \alpha 2(\mathrm{IX}) \alpha 3(\mathrm{IX})$ & $\begin{array}{c}\text { lateral association } \\
\text { with type II fibril }\end{array}$ & cartilage \\
\hline & $\mathrm{XII}$ & {$[\alpha .1(\mathrm{XII})]_{3}$} & $\begin{array}{c}\text { lateral association } \\
\text { with type I fibril }\end{array}$ & tendons, ligaments \\
\hline \multirow{2}{*}{$\begin{array}{l}\text { Network- } \\
\text { forming }\end{array}$} & IV & {$[\alpha 1(\mathrm{IV})]_{2} \alpha 2(\mathrm{IV})$} & $\begin{array}{l}\text { Sheet-like } \\
\text { network }\end{array}$ & basal lamina \\
\hline & VII & {$[\alpha 1(\mathrm{VII})]_{3}$} & anchoring fibrils & $\begin{array}{c}\text { beneath stratified squamous } \\
\text { epithelia }\end{array}$ \\
\hline
\end{tabular}


a b c

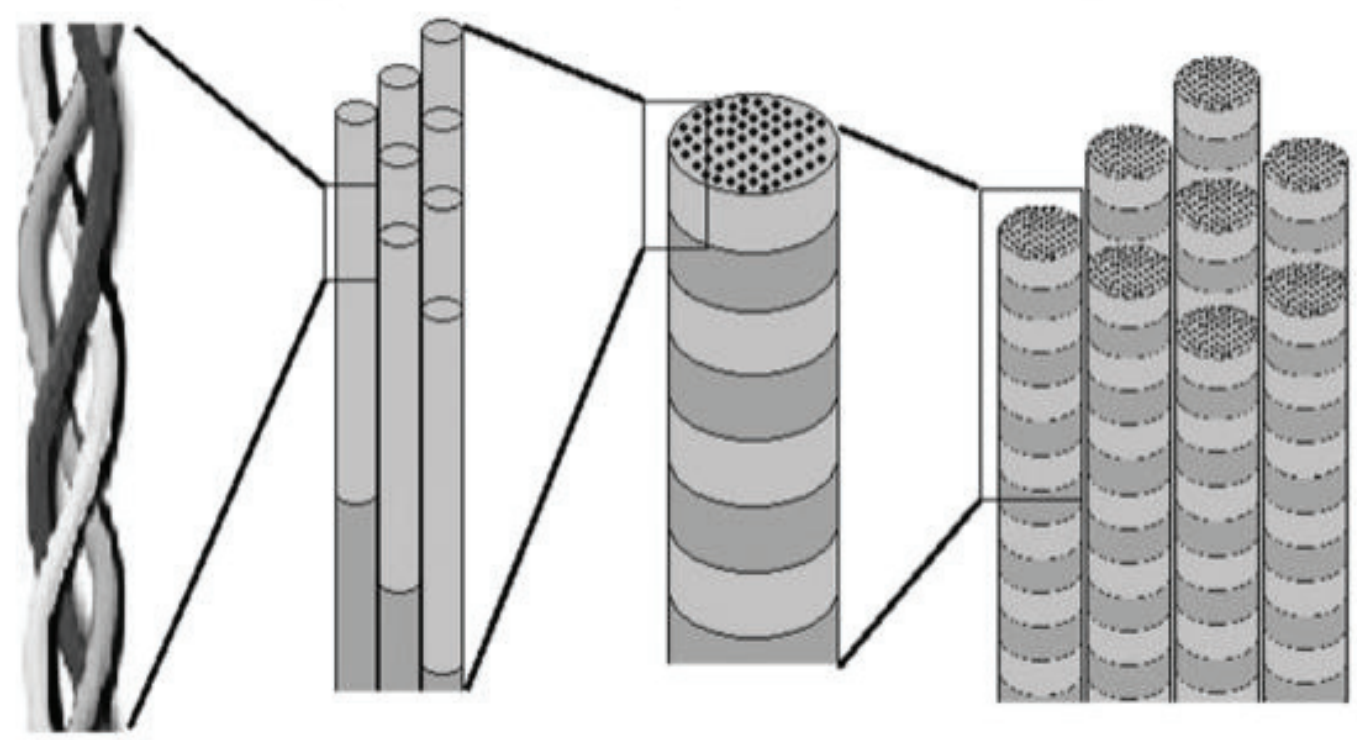

FIGURE 1. (a) Triple helix scheme of the collagen $\alpha$ chain. (B) Tropocollagen molecules are assembled. (c) Collagen fibrils with diameters ranging from 10 to $300 \mathrm{~nm}$. (D) Bundle of collagen fibrils form collagen fibers with diameters ranging from 0.5-3 $\mu \mathrm{m}[11]$.

\section{CHARACTERISTIC OF MARINE COLLAGEN}

More than half of the earth's surface is ocean. Marine species occupy half of global biodiversity, so marine species are an unlimited resource that provides collagen that can be utilized for medical needs, biomaterials, pharmaceutical products, food, and cosmetics. Because of the diversity of these marine species, the development and research of new sources of collagen for advanced biomedical applications $[12,13]$.

Marine vertebrates (fish and marine mammals), marine invertebrates (shrimp, sponges, jellyfish, squid, sea urchin, octopus, star fish, sea anemones, etc.), and other marine sources can be a source of marine collagen. Due to several beneficial properties including low immunogenicity, biocompatibility, high biodegradability, safety, water solubility, easy extraction but with high results, and low production costs, marine collagen has attracted wide scientific and industrial interest. Lots of fish waste is thrown away such as scales, guts, heads, bones, fins and skin. Finding adequate modalities to turn these leftover marine waste into useful products with high significance and economic value is important. Type I collagen is isolated from marine sources, usually used as an substantial biomaterial for scaffolding, while type IV and type II collagen can be extracted from some jellyfish, sea sponges, and fish cartilage [14, 15].

From all of the advantages, marine collagen has disadvantages. Almost all marine collagen contains lower amino acids, thus the denaturation temperature (Td) of marine collagen is lower than that of mammalian collagen. The difference in hydroxyproline might determine the $\mathrm{Td}$ of collagen. The water temperature of their normal habitat and their thermal stability is related with the amino acid content of fish collagens. Marine collagen melts at temperatures of more than $37^{\circ} \mathrm{C}$ because $\mathrm{Td}$ of marine collagen is lower than the body temperature of mammals.

Scaffold synthesized from marine collagen, mostly collagen from fish, has its own difficulties, such as lower Td and lower viscosity than terrestrial vertebrate collagen. Cross-linking in vitro can be done to improve collagen fibrillogenesis. Many cross-linking methods have been used to stabilize collagen. Cross-linking methods used to stabilize collagen can be divided into physical treatments, including gamma irradiation, UV irradiation, and dehydrothermal treatments and chemical treatments, such as those using the use of carbodiimide, glutaraldehyde, and ethyl dimethyl-aminopropyl carbodiimide (EDC). Chemical treatment provide very high strength and stability to the collagen matrix, whereas it can produce poor biocompatibility or potential cytotoxicity, whereas physical treatment provide sufficient stability without cytotoxicity [16-18]. 


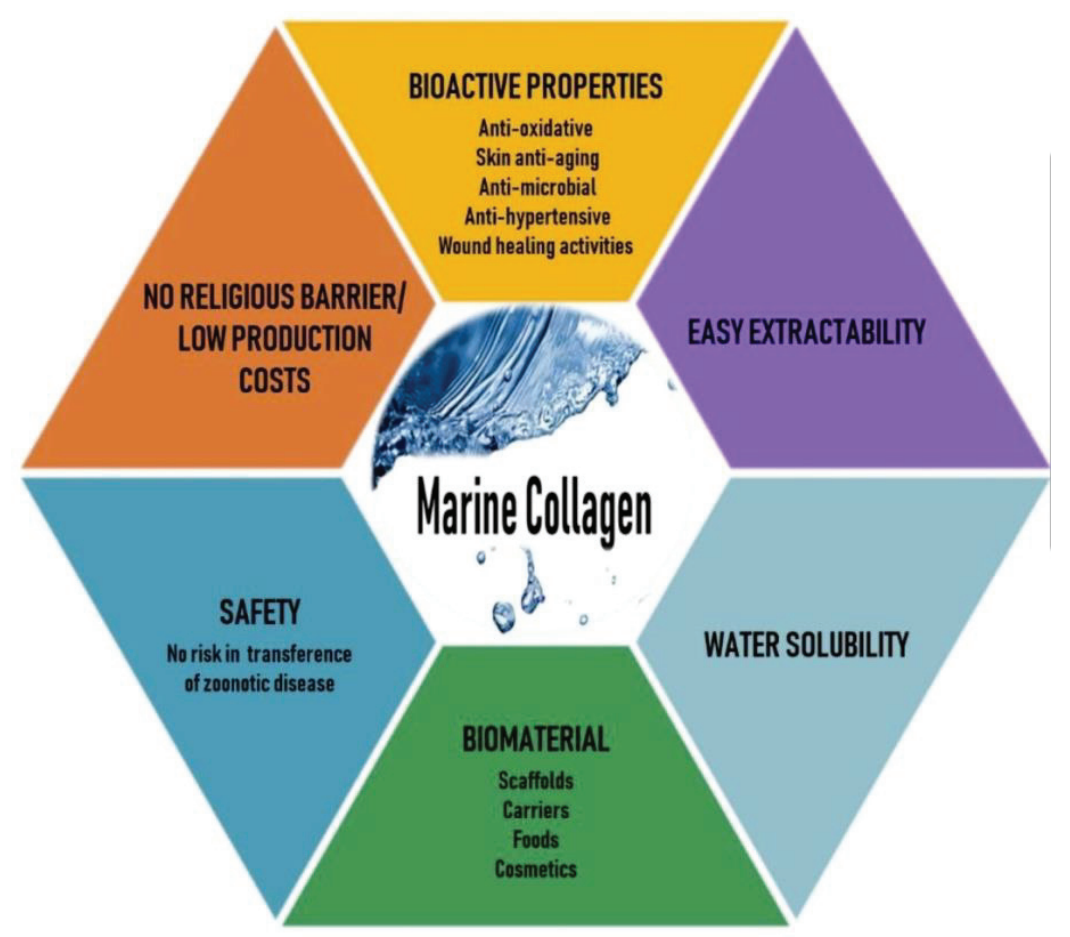

FIGURE 2. Advantages of marine source collagen [14].

\section{BIOCOMPATIBILITY}

The main reason for choosing collagen as a regenerative material is because collagen has low imunogenicity, biocompatibility, high biodegradability, and good direct cell adhesion compared to other natural and synthetic polymers. A tissue engineering experiment using fish collagen as scaffolding was carried out.

In a study of jellyfish collagen scaffolding carried out on cells cultured in a three-dimensional matrix, seeding of cells with high density and efficient nutrition and oxygen was provided because the collagen scaffold was very porous and the pores were interconnected. The ability of jellyfish collagen to stimulate an immune response comparable to bovine and / or gelatin collagen. Making collagen from tilapia to produce collagen sponge which has a microstructure is done by reconstructing collagen fibers using freeze dried and cross bonding through dehydrothermal (DHT). In an experimental study of pellet implantation in rabbit paravertebral muscle with negative control of porcine collagen and high density polyethylene it was found that tilapia collagen had almost the same statistical results. Where one or four weeks after implantation, collagen tilapia rarely triggers an inflammatory response [19-22].

\section{BIODEGRADATION}

In vitro degradation study concluded that the addition of freshwater fish scales collagen can provide a higher level of stability. In the study, scaffolding added with freshwater fish scales collagen was seen to decrease by $\sim 50 \%$ after 30 days, while scaffolding without addition experienced total degradation within 4 days. In studies conducted on mice injected with collagen and mice without treatment, it is known that collagen has minimal immunological reactions. It can be indicated that collagen biocompatible fish scales as tissue engineering in humans. Placement of graft material from marine collagen in subcutaneous mice is gradually biodegradable. One month after implantation, macrophages and fibroblasts penetrate the surface of the scaffold without symptoms of necrosis. In various types of treatment with collagen on implantation, no collagen is seen that disappears within one month after implantation [19-21]. 


\section{BIOMEDICAL APPLICATIONS}

\section{Tissue Engineering and Regeneration}

The development in the field of tissue engineering or regenerative medicine is very rapid, using biological techniques and principles to create new tissue and organ, to regenerate damaged tissues and organs by combining cells from the body with scaffold material. Bone tissue engineering studies require the selection of an appropriate scaffolding matrix. Excellent marine collagen biocompatibility is a potential role in tissue engineering and in regenerative medicine for the design of scaffold material. Most recently type I collagen is most widely used as a biomaterial. Chemical treatments provide very good strength and stability to the collagen matrix, despite they can produce poor biocompatibility or potential cytotoxicity, while physical treatment provide sufficient stability without cytotoxicity.

Biodegradable porous scaffolding applications that replicate natural extracellular matrix are needed for tissue engineering, which provides to spatially regulate cells and provide specific cellular signals and regulation. Important parameters that are widely recognized for scaffolding used in tissue engineering related to cell migration, seeding, growth, and formation of new tissue, surface area, number of pores, pore size, and pore wall morphology.

Polymer scaffolds directs various cellular processes based on structural and biochemical properties of scaffolds so that they have known as a central of tissue engineering technology. The material used to make scaffolds not only establishes the physical properties of mechanical stability, biocompatibility, and biodegradability, but also supplies the right signal to direct cellular processes that induce tissue formation. Collagen supports all types of connective tissues, including bones, cartilage, tendons, ligaments, skin, and blood vessels so it can be an ideal scaffold or carrier for tissue engineering.

Marine collagen has been researched as having osteogenic activity. Marine collagen extracted from tilapia scales can promote cell viability, significantly increase the expression of osteogenic and endothelial marker in the mesenchymal stem cell of the rat. It proves that marine collagen has the potential to promote osteogenic and endothelial differentiation. Interestingly, it also shows that marine collagen inhibits the expression of chondrogenic and adipogenic markers. Treatment with marine collagen increased the expression of osteogenic markers, activity of alkaline phosphatase, cell proliferation, and mineralization, which demonstrated the potential function of marine collagen as a biomaterial in osteoblast cell culture for tissue engineering in marine collagen extracted from bone and skin cod in human osteoblastic cell [23-26].

\section{Skin Tissue Engineering, Regeneration, and Wound Healing}

In clinical application, skin graft still has many limitations and weaknesses including donor availability. Due to increasing demand for skin replacement or skin defects caused by burns, trauma, infections, scarring, skin graft rejection, genetic defects and other diseases, this has become the major challenge for health care. There are three phases of the wound healing process in humans, namely: the inflammation phase, the proliferation phase and the maturation phase.

In the inflammatory phase, there is an increase in permeability to increase cell adhesion resulting in contraction at the wound edge during proliferation until maturation, regression and differentiation result in the appearance of new capillaries and differentiation in fibroblasts. And to continue the process of healing the wound, foreign particles and tissue that cannot survive must be immediately removed and cleaned from the wound surface. To increase the occurrence of homeostasis and to prevent infectious agents to penetrate, effective wound healing must be carried out [27-29].

The use of scaffold P (3HB-co-4HB) / marine collagen from tilapia skin increases the attachment of fibroblast proliferation and has a very significant effect in the process of wound healing and wound closure which shows that this scaffold can function as a wound healing technology in the future. The material used in this scaffold collagen is collagen obtained from mrigal fish (Cirrhinus cirrhosis) through the freeze drying method where the marine collagen sponge can stimulate proliferation and growth of primary fibroblasts and human keratinocyte when using this scaffold which causes faster wound healing and increases layers of the epidermis, proving that the use of this scaffold is proven as a skin substitute. Biocompatible scaffolding produced through Tilapia-scale collagen (Oreochromis niloticas) that form as hydrogel which is water soluble, can show that tilapia scales are a very effective source of collagen isolation used as ingredients that have potential to be used in skin regeneration and tissue engineering [32,34]. 


\section{Dental Tissue Engineering and Regeneration}

Marine collagen is responsible for osteogenic differentiation by increasing the viability of periodontal ligament cell and increasing the regulation of osteogenic markers and proteins associated with osteogenesis through ERK pathway. Marine collagen is a good biocative material as an alveolar guidance biomaterial. In clinical use, the tilapia collagen scaffold has the ability to enhance periodontal tissue repair by guiding tissue and bone repair membranes. In addition, the tilapia collagen membrane exhibits anti-bacterial properties against Streptococcus mutans and increases cell viability, cell adhesion, and osteogenic expression in periodontal ligament cell [34].

\section{CONCLUSION}

The selection of marine collagen as a biomaterial for tissue engineering because this collagen has good biocompability, soluble in water, safe, low-cost, easy to obtain, good biodegradation and antimicrobial. Marine collagen is natural and has a structure similar to mammalian collagen, so it has the potential as a biomedical biomaterial. Nevertheless, further experiments with experimental animals are needed before the material is used in humans.

\section{REFERENCES}

1. A. Silvestri, M. Boffito, S. Sartori, and G. Ciardelli, "Biomimetic materials and scaffolds for myocardial tissue regeneration," Macromolecular Bioscience, vol. 13, no. 8, pp. 984-1019, 2013.

2. Y.-C. Lin, F.-J. Tan, K. G. Marra, S.-S. Jan, and D.-C. Liu, "Syn- thesis and characterization of collagen/hyaluronan/chitosan composite sponges for potential biomedical applications," Acta Biomaterialia, vol. 5, no. 7, pp. 2591-2600, 2009.

3. M. H. Tayebjee, R. J. MacFadyen, and G. Y. H. Lip, "Extracellular matrix biology: a new frontier in linking the pathology and therapy of hypertension?" Journal of Hypertension, vol. 21, no.12, pp. 2211-2218, 2003.

4. Schmidt MM, Dornelles RCP, Mello RO, Kubota EH, Mazutti MA, Kempka AP, et al. Collagen extraction process. Int Food Res J. 2016;23(3):913-22.

5. X. Wang, C. You, X. Hu et al., "The roles of knitted mesh- reinforced collagen-chitosan hybrid scaffold in the one-step repair of full-thickness skin defects in rats," Acta Biomaterialia, vol. 9, no. 8, pp. 7822-7832, 2013.

6. N. Kawaguchi, K. Hatta, and T. Nakanishi, "3D-culture system for heart regeneration and cardiac medicine," BioMed Research International, vol. 2013, Article ID 895967, 6 pages, 2013.

7. Y. Hayashi, S. Yamada, T. Ikeda, Z. Koyama, and K. Yanagiguchi, "Chitosan and fish collagen as biomaterials for regenerative medicine," in Marine Medical Food, S.-K. Kim, Ed., vol. 65, chapter 6, pp. 107-120, Academic Press, London, UK, 2012.

8. A. K. Dillow and A. M. Lowman, Biomimetic Materials and Design: Biointerfacial Strategies, Tissue Engineering, and Tar- geted Drug Delivery, Mercel Dekker, New York, NY, USA, 2002.

9. S. Yang, K.-F. Leong, Z. Du, and C.-K. Chua, "The design of scaffolds for use in tissue engineering. Part I. Traditional factors," Tissue Engineering, vol. 7, no. 6, pp. 679-689, 2001.

10. K. Yamamoto, Y. Yoshizawa, K. Yanagiguchi, T. Ikeda, S.

11. Yamada, and Y. Hayashi, "The characterization of fish (tilapia) collagen sponge as a biomaterial," International Journal of Polymer Science, vol. 2015, Article ID 957385, 5 pages, 2015.

12. Parenteau-Bareil R, Gauvin R, Berthod F. Collagen-based biomaterials for tissue engineering applications. Materials (Basel). 2010;3(3):1863-87.

13. Kim, J.A.; Kim, H.N.; Im, S.K.; Chung, S.; Kang, J.Y.; Choi, N. Collagen-based brain microvasculature model in vitro using three-dimensional printed template. Biomicrofluidics 2015, 9, 024115.

14. Pallela, R.; Ehrlich, H. Marine Sponges: Chemicobiological and Biomedical Applications; Springer: New Delhi, India, 2016; ISBN 978-81-322-2792-2.

15. Malve, H. Exploring the ocean for new drug developments: Marine pharmacology. J. Pharm. Bioallied Sci. 2016, 8, 83-91.

16. Venkatesan, J.; Anil, S.; Kim, S.K.; Shim, M.S. Marine Fish Proteins and Peptides for Cosmeceuticals: A Review. Mar. Drugs 2017, 15, 143.

17. Muyonga, J.H.; Colec, C.G.B.; Duodub, K.G. Extraction and physicochemical characterisation of Nile perch (Lates niloticus) skin and bone gelatine. Food Hydrocoll. 2004, 18, 581-592. 
18. Nomura, Y.; Sakai, H.; Ishii, Y.; Shirai, K. Preparation and some properties of type I collagen from fish scales. Biosci. Biotechnol. Biochem. 1996, 60, 2092-2094.

19. Foegeding, E.A.; Lanier, T.C.; Hultin, H.O. Food Chemistry; Marcel Dekker: New York, NY, USA, 1996; Chapter 15; pp. 902-924. ISBN 0-8247-9346-3

20. F. Pati, P. Datta, B. Adhikari, S. Dhara, K. Ghosh, and P. K.D. Mohapatra, "Collagen scaffolds derived from fresh water fish origin and their biocompatibility," Journal of Biomedical Materials Research Part A, vol. 100, no. 4, pp. 1068-1079, 2012.

21. C. H. Lee, A. Singla, and Y. Lee, "Biomedical applications of collagen," International Journal of Pharmaceutics, vol. 221, no.1-2, pp. 1-22, 2001.

22. N. Nagai, Y. Nakayama, Y.-M. Zhou, K. Takamizawa, K. Mori, and M. Munekata, "Development of salmon collagen vascular graft: mechanical and biological properties and preliminary implantation study," Journal of Biomedical Materials Research Part B, vol. 87, no. 2, pp. 432-439, 2008.

23. H. Sugiura, S. Yunoki, E. Kondo, T. Ikoma, J. Tanaka, and K. Yasuda, "In vivo biological responses and bioresorption of tilapia scale collagen as a potential biomaterial," Journal of Biomaterials Science, Polymer Edition, vol. 20, no. 10, pp. 1353-

24. 1368, 2009.

25. Yamada, S.; Yoshizawa, Y.; Kawakubo, A.; Ikeda, T.; Yanagiguchi, K.; Hayashi, Y. Early gene and protein expression associated with osteoblast differentiation in response to fish collagen peptides powder. Dent. Mater. J. 2013, 32, 233-240.

26. G. Chen, T. Ushida, and T. Tateishi, "Scaffold design for tissue engineering," Macromolecular Bioscience, vol. 2, pp. 67-77, 2002.

27. S. Yang, K.-F. Leong, Z. Du, and C.-K. Chua, "The design of scaffolds for use in tissue engineering. Part I. Traditional factors," Tissue Engineering, vol. 7, no. 6, pp. 679-689, 2001.

28. B. L. Seal, T. C. Otero, and A. Panitch, "Polymeric biomaterials for tissue and organ regeneration," Materials Science and Engi- neering: R: Reports, vol. 34, no. 4-5, 2001.

29. Sitje, T.S.; Grøndahl, E.C.; Sørensen, J.A. Clinical innovation: Fish-derived wound product for cutaneous wounds. Wounds Int. 2018, 9, 44-50.

30. Wolcott, R.; Fletcher, J. The role of wound cleansing in the management of wounds. Wounds Int. 2014, 1, 2531.

31. Elias, P.M. The skin barrier as an innate immune element. Semin. Immunopathol. 2007, 29, 3-14.

32. Menon, G.K.; Kligman, A.M. Barrier functions of human skin: A holistic view. Skin Pharmacol. Physiol. 2009, 22, 178-189.

33. Vigneswari, S.; Murugaiyah, V.; Kaur, G.; Abdul Khalil, H.P.; Amirul, A.A. Biomacromolecule immobilization: Grafting of fish-scale collagen peptides onto aminolyzed $\mathrm{P}(3 \mathrm{HB}-\mathrm{co}-4 \mathrm{HB})$ scaffolds as a potential wound dressing. Biomed. Mater. 2016, 11, 055009.

34. Pal, P.; Srivas, P.K.; Dadhich, P.; Das, B.; Maity, P.P.; Moulik, D.; Dhara, S. Accelerating full thickness wound healing using collagen sponge of mrigal fish (Cirrhinus cirrhosus) scale origin. Int. J. Biol. Macromol. 2016, 93, $1507-1518$.

35. El-Rashidy, A.A.; Gad, A.; Abu-Hussein, A.H.; Habib, S.I.; Badr, N.A.; Hashem, A.A. Chemical and biological evaluation of Egyptian Nile Tilapia (Oreochromis niloticas) fish scale collagen. Int. J. Biol. Macromol. 2015, 79, $618-626$.

36. Ye-Seon Lim,Ye-Jin Ok ,Seon-Yeong Hwang ,Jong-Young Kwak and Sik Yoon. Marine Collagen as A Promising Biomaterial for Biomedical Applications. Mar. Drugs 2019, 17(8), 467 